\title{
因HAD
}

ISSN-L: 2530-5115

(c) (1) (2)

DOI: http://doi.org/10.22585/hospdomic.v4i2.97

\section{Dolor irruptivo oncológico de mal control secundario a masas suprarrenales bilaterales}

\section{Breaktrhough cancer pain bad controlled secondary to bilateral adrenal masses}

Asier Díaz Esteban', Selene Illán Varella², Andrea Illán Varella³, Sonia Panadero Amado'

1. Hospital San Pedro de Alcántara, Hospitalización Oncología y Hematología, Cáceres, España.

2. Hospital General Universitario, Hospitalización Oncología y Hematología, Alicante, España.

3. Hospital Virgen del Puerto, Servicio de Oncología Médica, Plasencia, España.

Correspondencia/Correspondence

Asier Díaz Esteban

Email: asierdmb@gmail.com

Recibido/Received

12.01 .2020

Aceptado/Accepted

16.03.2020
Conflicto de Intereses/Competing interest Los autores no declaran ningún tipo de conflicto de intereses.

CÓMO CITAR ESTE TRABAJO | HOW TO CITE THIS PAPER

Díaz A, Illán S, Illáns A, Panadero S. Dolor irruptivo oncológico de mal control secundario a masas suprarrenales bilaterales. Hosp Domic. 2020;4(2):89-94. 


\section{RESUMEN}

Presentamos el caso de un paciente varón de 67 años diagnosticado de adenocarcinoma de pulmón en estadio IV con dolor irruptivo oncológico (DIO) de difícil control. Durante el transcurso de la enfermedad el paciente necesitó varios ingresos por complicaciones, siendo la más común el mal control del DIO. Tras varios cambios en la pauta analgésica (rotación de opioides, cambios en tratamientos coadyuvantes y vías de administración), se consiguió buen control del dolor a pesar de progresión de la enfermedad de base. Finalmente, el paciente precisó ingreso hospitalario ante deterioro del estado general y claudicación familiar. Ante mal pronóstico, el equipo de Cuidados Paliativos y los familiares decidieron instaurar tratamiento con medidas de confort. De la relevancia del caso clínico que aportamos se concluye que es necesaria la existencia de equipos multidisciplinares formados y entrenados en este ámbito para un buen manejo terapéutico y una mejor calidad de vida en los pacientes.

Palabras clave: Dolor Irruptivo; Neoplasias de las Glándulas Suprarrenales; Metástasis de la Neoplasia; Dolor; Dolor en Cáncer; Neoplasias Pulmonares.

\section{ABSTRACT}

We present the case of a 67-year-old male with stage IV adenocarcinoma lung cancer who presented difficult control of breakthrough cancer pain. During the course of the disease, the patient had to be hospitalized several times due to complications. The most common complication was breakthrough cancer pain. After many changes in the analgesic treatment (opioid rotation, changes in coadjuvant drugs and routes of administration), good control of analgesic pain was achieved despite the progression of this underlying disease. Finally, the patient required being hospitalized due to deterioration of the general condition and family claudication. Faced with a por prognosis, the Palliative Care and Oncology team decided to establish treatment with comfort measures. We can conclude that the existence of multidisciplinary trained team is necessary for good therapeutic management and a better quality of life in patients.

Keywords: Breakthrough Pain; Adrenal Gland Neoplasms; Neoplasm Metastasis; Dolor; Cancer Pain; Lung Neoplasms. 


\section{INTRODUCCIÓN}

Nos disponemos a realizar la descripción de un caso clínico consistente en un paciente varón de 67 años diagnosticado de adenocarcinoma de pulmón estadio IV en base a masas metastásicas suprarrenales bilaterales que presentó dolor irruptivo oncológico espontáneo de difícil control.

Como es sabido, uno de los síntomas principales del cáncer, especialmente en situaciones avanzadas, es el dolor(1,2). La Asociación Internacional para el Estudio del Dolor (IASP) define el dolor como aquella experiencia sensorial o emocional desagradable asociada a un daño real o potencial en un tejido, o descrito en términos de dicho daño(1,3). El dolor oncológico, por tanto, se acepta cuando dicha experiencia sensorial o emocional es secundaria al propio tumor o al tratamiento anticanceroso(2).

La escalera terapéutica del dolor de la Organización Mundial de la Salud (OMS) de 1996 es la herramienta que se utiliza para el tratamiento de este síntoma en el paciente oncológico. Se fundamenta en el uso de medicación analgésica de forma progresiva; empezando por fármacos no opioides ( $1^{\text {er }}$ escalón) y ultimando en opioides ( $2^{\circ}$ y $3^{\text {er }}$ escalón) e incluso con técnicas intervencionistas ( $4^{\circ}$ escalón) según las necesidades de cada paciente. Sin embargo, en ocasiones, es necesario utilizar directamente opioides potentes para conseguir un buen control del dolor; fenómeno que se conoce como "ascensor analgésico"(4).

El dolor irruptivo oncológico (DIO) se define como una exacerbación aguda y transitoria de dolor de aparición rápida, corta duración y de moderada a elevada intensidad(5) que aparece de forma espontánea o por un desencadenante concreto, predecible o impredecible, a pesar de existir un dolor basal estable y adecuadamente controlado(1). Su frecuencia de aparición es variable, de una a varias veces al día. La prevalencia en pacientes con cáncer es alta (23-93\%)(4,5), por lo que se evidencia una problemática clínica notable. Este síntoma de difícil manejo(3), si está mal controlado, da como resultado cuadros de ansiedad y depresión, interfiere en el sueño y en la vida laboral, personal y social, disminuyendo así la calidad de vida del paciente.

Actualmente, el fentanilo transmucoso(4) por vía intranasal es el analgésico de elección para tratar el dolor irruptivo en este tipo de pacientes porque además de ser un opioide potente, sus características se ajustan al que sería el fármaco ideal para el tratamiento de las crisis de DIO. Nos referimos a un inicio de acción rápido, una alta potencia farmacológica, una vida media corta y que, a parte, es autoadministrable.

Nos disponemos a describir nuestro caso clínico para valorar la importancia de un DIO de manejo complejo.

\section{Desarrollo de la experiencia clínica}

Presentamos el caso clínico de un paciente varón de 67 años, con hábitos tóxicos de fumador de 50 paquetes/año y bebedor de alcohol ocasional y sin otros antecedentes de interés.

El paciente inició estudio en base a cuadro de 6 meses de evolución de dolor continuo localizado en hipogastrio e irradiado en miembros inferiores (MMII). Relataba también anorexia y pérdida de $10 \mathrm{~kg}$ de peso en los últimos 3 meses. Se realizó resonancia magnética nuclear (RMN) abdominal y tomografía axial computerizada (TAC) toraco-abdomino-pélvica, que pusieron de manifiesto los siguientes hallazgos:

1. Masa con corona radiata en lóbulo superior derecho (segmento I) que de dimensiones 55×30×65 mm de diámetros AP transverso y craneocaudal respectivamente, con contacto con el bronquio del LSD (sin obliteración) y con la pleura visceral. 
2. A nivel adrenal bilateral, masas hipercaptantes, la derecha de dimensiones de $76 \times 38 \times 40 \mathrm{~mm}$ y la izquierda de $63 \times 37 \times 55$, sugerentes de localizaciones metastásicas.

Ante los hallazgos de las pruebas de imagen, se realizó broncoscopia diagnóstica, con anatomía-patológica (A-P) de la biopsia bronquial compatible con adenocarcinoma poco diferenciado. Desde Medicina Interna (quien realizó estudio) se reajustó analgesia en varias ocasiones, debido a dolor irruptivo intenso sin desencadenante de duración inferior a una hora localizado en flanco derecho con irradiación a la región dorsal y EVA 8/10, pautándole finalmente: Morfina de liberación sostenida 30 mg cada 12 h vo, Dexametasona 4 mg cada 24 h vo, Morfina de liberación rápida 10 mg de rescate vo.

El paciente fue derivado a consultas externas de Oncología Médica para valoración de tratamiento. Se planteó tratamiento de primera línea según esquema Carboplatino-Pemetrexed, a la espera de obtención de biomarcadores (EGFR, ALK, ROS1, PDL-1). Antes de la administración del primer ciclo, el paciente ingresó en planta de Oncología Médica con juicios clínicos de: insuficiencia suprarrenal secundaria a infiltración metastásica de las ambas glándulas, hiperpotasemia grave resuelta, hiponatremia moderada, fracaso renal agudo, fiebre sin claro foco en paciente oncológico y mal control del dolor oncológico. Respecto a este último punto, fue precisa rotación de opioides a vía intravenosa por mal control de dolor (múltiples episodios de dolor irruptivo oncológico (DIO) de tipo nociceptivo visceral de aparición espontánea, gradados por EVA como 10/10, localizados en ambos flancos manejados con mórfico parenteral) y posterior cambio a la vía oral.

Se pautó primer ciclo de Carboplatino-Pemetrexed sin incidencias agudas.

El paciente fue dado de alta a domicilio con el siguiente tratamiento analgésico: Morfina de liberación sostenida $40 \mathrm{mg}$ vo cada 12 horas, Dexametasona 4 mg vo 1-1/2-0 comp (y posterior pauta descendente), Paracetamol o Metamizol 1 comp vo cada 8 h (si dolor leve), Fentanilo de liberación ultrarrápida sublingual $100 \mathrm{mcg}$ (si dolor intenso).

El paciente consultó a su MAP por dolor irruptivo de las mismas características citadas anteriormente que reaparecía rápidamente tras la toma de Fentanilo ultrarrápido sublingual 200 mcg de rescate (tras proceso de titulación adecuado). Le fue pautado Clonixino lisina, que tomaba cada 8 h vo, experimentando gran mejoría del control del dolor.

Ya en consultas externas, el paciente mantenía el control del dolor irruptivo, puntuándolo con EVA 4/10. A nivel oncológico, se obtuvo resultado de PDL-1 en la muestra de la broncoscopia, teniendo un status positivo del $90 \%$; por ello, se dio finalizado tratamiento quimioterápico y se sustituyó por inmunoterapia basada en Pembrolizumab.

El paciente presentó dos ingresos posteriores por mal control del dolor. El paciente presentó $>6$ episodios de DIO diarios, que no manejaba adecuadamente pese a fentanilo de rescate y los otros fármacos de primer y tercer escalón. Se solicitó durante el ingreso TC abdominal, que confirmó pseudoprogresión, por lo que fue precisa la reevaluación precoz radiológica a las 4 semanas. Dicho TC confirmó progresión oncológico real con aumento del tamaño de las metástasis suprarrenales hasta $78 \mathrm{~mm}$ de diámetro mayor bilateralmente.

Durante el ingreso, precisó nueva rotación opioide a morfina intravenosa; y posteriormente, desde la vía parenteral hasta la vía transdérmica con Fentanilo 75 mcg cada 72 h, con aceptable control del dolor. Para el control del DIO de intensidad muy elevada, se mantuvo Clonixino lisina (el paciente insistía en que era muy efectivo) seguido de morfina de liberación rápida administrada de forma parenteral (ya que el paciente refería que el fentanilo de liberación rápida tenía efecto, pero con duración escasa pese a titulación adecuada; y ya que morfina de liberación rápida (no había demostrado ser efectivo con anterioridad) de $20 \mathrm{mg}$ vo. Mantuvo como coadyuvantes y analgesia de primer escalón con: Lorazepam 1 mg: 1/2-1/2-1, Dexametasona 4 mg vo: 1-0-0, Paracetamol 1 g vo: 1-1-1; Le fue pautado Clonixino lisina, que tomaba cada $8 \mathrm{~h}$ vo, experimentando gran mejoría del control del dolor. 
El paciente experimentó mejoría en el control del dolor, pero deterioro progresivo del estado general. Se hizo interconsulta a la Unidad de Cuidados Paliativos (UCP), para seguimiento y tratamiento sintomático en el domicilio (se consensuó con el paciente y familiar alta al mismo ante situación de últimos días). A partir de dicho momento, el paciente se mantuvo con medicación oral estrictamente necesaria (el paciente presentaba hiporexia y escasa ingesta hídrico-alimenticia) y como rescate se mantuvo el mórfico por vía subcutánea (el paciente solicitaba 2 rescates diarios por DIO) así como sueroterapia (500 cc suero salino fisiológico cada 24 horas). UCP supervisó a la familiar en la pauta de los rescates y realizó contacto telefónico y una visita en el domicilio.

Unos días más tarde, el paciente reingresó por claudicación familiar. El paciente mostraba gran deterioro del estado general, caquexia y negativa completa a la ingesta en los últimos días. Además, inició cuadro de disnea con pequeños esfuerzos. La Rx de tórax al ingreso demostró atelectasia de lóbulo superior derecho (LSD) e infiltrado neumónico de lóbulo medio derecho (LMD) compatible con neumonía infecciosa.

Ante los signos y síntomas de situación de últimos días y el pronóstico infausto del paciente, se consensuó con la familia medidas de confort exclusivas. El paciente fue exitus letalis a las 48 horas del ingreso.

\section{CONCLUSIONES}

La prevalencia del dolor en el paciente oncológico al diagnóstico es del 30-50\% y se incrementa hasta el $90 \%$ en estadios avanzados de la enfermedad. Por otra parte, literatura reciente expone que hasta un 50\% de pacientes tienen mal control de su dolor oncológico a pesar de la disponibilidad de tratamientos eficaces que hay en la actualidad. La intensidad es moderada-severa en más de la mitad de los pacientes y es considerado como el síntoma más invalidante que afecta a la calidad de vida de los pacientes. Por ello, se necesitan equipos entrenados en el manejo de los múltiples tratamientos para el control del dolor oncológico, y especialmente de tipo irruptivo, sin olvidarse de la importancia de la empatía en dichos profesionales con el fin de llevar a cabo un abordaje adecuado.

\section{BIBLIOGRAFÍA}

1. Van den Beuken-van Everdingen MH, de Rijke JM, Kessels AG, Schouten HC, van Kleef M, Patijn J. Prevalence of pain in patients with cancer: a systematic review of the past 40 years. Ann Oncol 2007;18(9):1437-49. DOI: 10.1093/annonc/mdm056; PMID: 17355955

2. Bennett MI, Kaasa S, Barke A, Korwisi B, Rief W, Treede RD, et al; IASP Taskforce for the Classification of Chronic Pain. The IASP classification of chronic pain for ICD-11: chronic cancer-related pain. Pain. 2019;160(1):38-44. DOI: 10.1097/j.pain.0000000000001363; PMID: 30586069

3. Devonshire E, Nicholas MK. Continuing education in pain management: using a competency framework to guide professional development. Pain Rep. 2018;3(5):e688. DOI: 10.1097/ PR9.0000000000000688; PMID: 30534629

4. Caraceni A, Shkodra M. Cancer pain assessment and classification. Cancers (Basel). 2019;11(4). pii:E510. DOI: 10.3390/cancers11040510; PMID: 30974857 
Notas Clínicas: Díaz A, Illán S, Illáns A, Panadero S

5. Escobar Álvarez Y, Biete i Solá A, Camba Rodríguez M, Galvez Mateos R, Mañas Rueda A, Rodríguez Sánchez CA, et al. Diagnóstico y tratamiento del dolor irruptivo oncológico: recomendaciones de consenso. Rev Soc Esp Dolor 2013;20(2):61-8. DOI: 10.4321/S113480462013000200005

6. Bruera E, Portenoy RK. Cancer Pain. 2nd edition. Cambridge, UK: Cambridge University Press; 2010. p. 37-52. 